one finck $\lambda^{(1)}=0.45016, \lambda^{(2)}=0.49818, \lambda^{(3)}=0.49988$, etc. The convergence of the sequence $; \lambda^{(p)}$; to the true value $\nu_{1}=\frac{1}{2}$ is strongly suggested.

Department of Engineering Mechanies

University of Michigan

1. Bholaxath PaL, "(On the numerical calculation of the roots of the equations $P_{n}{ }^{m}(\mu)=0$ and $\left(d /(l \mu) P^{\prime}{ }_{n}{ }^{\prime}(\mu)=0\right.$, regarded as equations in $n, "$ Bull. Calcutta Math. Soc., v. 9, 1917-1918, pp. 85-96.

2. BHOLANATH PAL, "On the numerical calculation of the roots of the equations $P_{n}{ }^{m}(\mu)=0$ and $(d / d \mu) P_{n}{ }^{m}(u)=0$, regarded as equations in $n$," Bull. Calcutta Math. Soc., v. 10, 1918-1919, pp. 187-194.

3. Wilhelm Magnus \& Fritz Oberhettinger, Formeln und Sätze für die speziellen Funktionen der mathematischen Physik, Springer, Berlin, 1943; 2nd ed., 1948; English transl., Chelsea, New York, 1949. MR 9, 183; MR 10, 38, 532.

\title{
Some Integrals of Ramanujan and Related Contour Integrals*
}

\author{
By Van E. Wood
}

1. Introduction. The integrals

$$
I_{n}{ }^{k}(t)=(2 \pi i)^{-1} \int_{-\infty}^{(0+)} e^{z t} z^{n-1}(\ln z)^{k} d z, \quad \operatorname{Re} t>0
$$

occur in the asymptotic expansions of the solutions of heat conduction problems in regions bounded internally by a circular cylinder [1], in problems on the flow of fluids through porous media [2], in electron slowing-down problems [3], and elsewhere. It should be recognized that these integrals are not in general the inverse Laplace transforms of $z^{n-1}(\ln z)^{k}$, since the contour does not surround the singularity occurring at $z=1$ when $k<0$. We will consider only cases where $t$ is real and $n$ and $k$ are integers. For $k$ nonnegative, the integrals can be expressed in terms of polygamma functions [2]. For nonnegative $n$ and negative $k$, they can be expressed, by means of change of variables and integrations by parts, in terms of derivatives of Ramanujan's integral [4],

$$
I_{R}(t)=\int_{0}^{\infty} e^{-t x} x^{-1}\left(\pi^{2}+\ln ^{2} x\right)^{-1} d x .
$$

This function is in turn related to the $\nu$-functions of Volterra and others [5, 6], which are useful in the solution of certain integral equations. In this paper, we discuss properties and numerical values of Ramanujan's integral, its derivatives, and the related contour integrals.

2. Relation to Other Integrals. Using the recurrence relations

$$
d I_{n}{ }^{k}(t) / d t=I_{n+1}^{k}(t)
$$

Received Oct ober $(6,1965$.

* This work was supported in part by the U. S. Air Force Office of Scientific Research Grant No. AF-AFOSR-262-63. 
and

$$
t I_{1}^{k}(t)=-k I_{0}^{k-1}(t),
$$

the $I_{n}{ }^{k}$ integrals with $k<0$ of most common occurrence are easily related to integrals along the positive real axis as follows:

$$
\begin{aligned}
& -I_{0}^{-1}=I_{R} \\
& -I_{0}^{-2}=t I_{R}^{(1)} \\
& -I_{1}^{-1}=I_{R}^{(1)} \\
& -I_{1}^{-2}=I_{R}^{(1)}+t I_{R}^{(2)} \\
& -I_{2}^{-1}=I_{R}^{(2)} \\
& -I_{2}^{-2}=2 I_{R}^{(2)}+t I_{R}^{\left({ }^{(3)}\right.} \\
& -2 I_{2}^{-3}=4 I_{R}^{(2)}+5 t I_{R}^{(3)}+t^{2} I_{R}{ }^{(4)}
\end{aligned}
$$

where the arguments have been suppressed, and $I_{R}{ }^{(n)}$ indicates, the $n$th derivative of $I_{R}$.

The derivatives of $I_{R}$ may be expressed in terms of the "overcomplete" $\nu$-functions [6] according to the relation discovered by Ramanujan [4]

$$
I_{R}^{(n)}(t)=e^{t}-\int_{-n}^{\infty} \frac{t^{x} d x}{\Gamma(1+x)} .
$$

(Ramanujan in fact proved the more general identity

$$
\int_{-\xi}^{\infty} \frac{t^{x}}{\Gamma(1+x)} d x+\int_{0}^{\infty} x^{\xi-1} e^{-t x}\left(\cos \pi \xi-\frac{\sin \pi \xi}{\pi} \ln x\right) \frac{d x}{\pi^{2}+\ln ^{2} x}=e^{t} .
$$

Hardy [4] states that the conditions for the validity of this formula are $t \geqq 0, \xi \geqq 0$. The conditions should actually be $t>0, \xi \geqq 0$ and $t=0, \xi=0$.) From this relation we may derive the result

$$
e^{-t} I_{R}(t)=\int_{0}^{1} d y \Gamma(y, t) / \Gamma(y)
$$

where $\Gamma(y, t)$ is an incomplete gamma function [7].

3. Asymptotic Expansions. Ritchie and Sakakura [2] have given asymptotic expansions for the integrals $I_{n}{ }^{k}$, while an expansion for $I_{R}$ was given by Spencer and Fano [3]. By the method of the latter article we may obtain asymptotic expansions for the derivatives of $I_{R}$ having the form

$$
(-t)^{n} I_{R}{ }^{(n)}(t) \sim \frac{\Gamma(n)}{\pi^{2}+\ln ^{2} t} \sum_{i=0}^{\infty} \frac{(i+1) A_{n ; i}(t)}{\left(\pi^{2}+\ln ^{2} t\right)^{i}} .
$$

The first few coefficients $A_{n ; i}(t)$ are given in Table I.

4. Numerical Values. In lieu of presenting extensive tables of values, we give in this section some graphs and a discussion of methods of calculation of $I_{R}$ and its derivatives. A discussion of approximations is given in the next section. 
TABLE I

\begin{tabular}{lc} 
('ocfficients in as!ymptotic expansion "f $t^{n} I_{R}{ }^{(n)}(l)$ \\
$i$ & $A_{n: i}(t)$ \\
0 & 1 \\
1 & $\psi(n) \ln l$ \\
2 & $\left(\psi^{2}(n)+\psi^{(1)}(n)\right)\left(\pi^{2} / 3+\ln ^{2} t\right)$ \\
3 & $\left(\psi^{3}(n)+3 \psi(n) \psi^{(1)}(n)+\psi^{(2)}(n)\right) \ln ^{3} t$ \\
& $\psi(z) \equiv d \ln \mathrm{\Gamma}(z) / d z ; \quad \psi^{(j)}(z) \equiv d^{j} \psi(z) / d z^{j}$. \\
\hline
\end{tabular}

The most straightforward way of calculating $I_{\boldsymbol{R}}(t)$ for any argument seems to be: (i) change the variable of integration to $v=t x$, (ii) split the range of integration into two parts, and (iii) eliminate the singularity in the integrand at the origin by the transformation $e^{-v} \rightarrow 1+\left(e^{-v}-1\right)$. Thus we have

$$
\begin{aligned}
I_{R}(t) & =I_{R 1}+I_{R 2}+I_{R 3} ; \\
I_{R 1} & =\frac{1}{2}-\pi^{-1} \tan ^{-1}((\ln 2 t) / \pi) ; \\
I_{R 2} & =-\int_{0}^{1 / 2} \frac{\left(1-e^{-v}\right)}{\left(\pi^{2}+\ln ^{2}(v / t)\right)} \frac{d v}{v} ; \\
I_{R 3} & =\int_{1 / 2}^{\infty} \frac{e^{--v}}{\left(\pi^{2}+\ln ^{2}(v / t)\right)} \frac{d v}{v} .
\end{aligned}
$$

The range of integration is split at $v=\frac{1}{2}$ only because this value seems to work fairly well; no attempt has been made to optimize this parameter to a high degree of accuracy. The term $I_{R 3}$ may be obtained by Gauss-Laguerre quadrature, 32-point quadrature [8] giving at least 6 decimal place accuracy, while $I_{R 2}$ is calculable by a brute-force method, such as using Gauss-Legendre quadrature in successively smaller subintervals until the desired accuracy is obtained. Figure 1 is a graph of $I_{R}(t)$.

The derivatives of $I_{R}$ (multiplied by an appropriate power of $t$ ) may be calculated in a similar way; however, since there is no longer a singularity in the integrand, one may consider the accuracy of a 32-point Gauss-Laguerre quadrature over the entire range of the integral. It turns out that one may obtain $t I_{R}{ }^{(1)}$ correct to 4 decimal places this way, and the higher functions $t^{n} I_{R}{ }^{(n)}$ correct to 5 d.p. Curves of $(-t)^{n} I_{R}{ }^{(n)}(t) / \Gamma(n)$ for $n=1,2,3,4$ are shown in Figure 2.

Values of those contour integrals $I_{n}{ }^{k}(t)$ which are not trivially related to functions already graphed are shown in Figure 3.

5. Approximations. Because of the occurrence of infinite derivatives at $t=0$, it is not particularly easy to find simple rational approximations to these functions useful over the entire range of $t$. By excluding a small region near the origin, however, one may obtain reasonably accurate approximations; for instance,

$$
I_{R 2}+I_{R 3} \approx \frac{\left(u-u_{0}\right) \sum_{i=0}^{3} a_{i} u^{i}}{\left(\pi^{2}+u^{2}\right)\left(u+\frac{1}{2} u_{0}\right) \sum_{i=0}^{2} b_{i} u^{i}},
$$




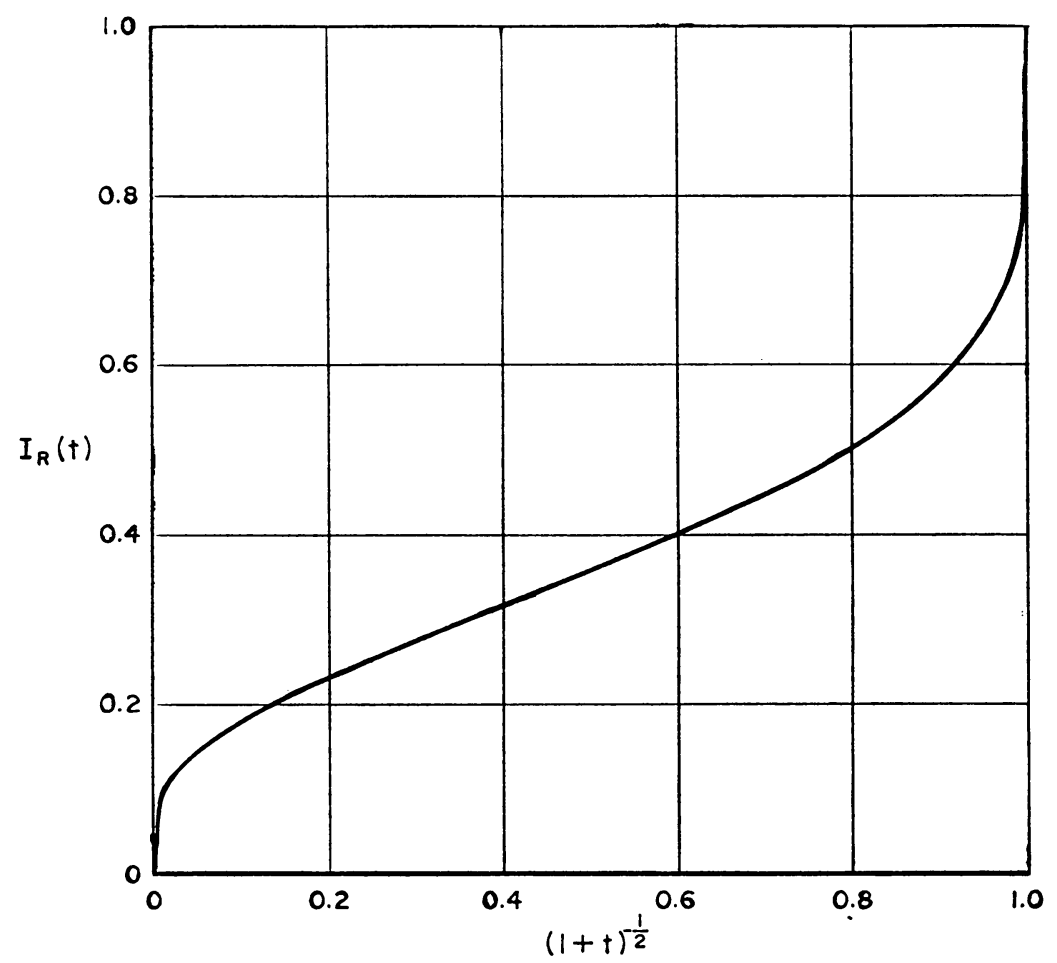

Fig. 1. Ramanujan's integral $I_{R}(t)=\int_{0}^{\infty} e^{-t x} x^{-1}\left(\pi^{2}+\ln ^{2} x\right)^{-1} d x$.

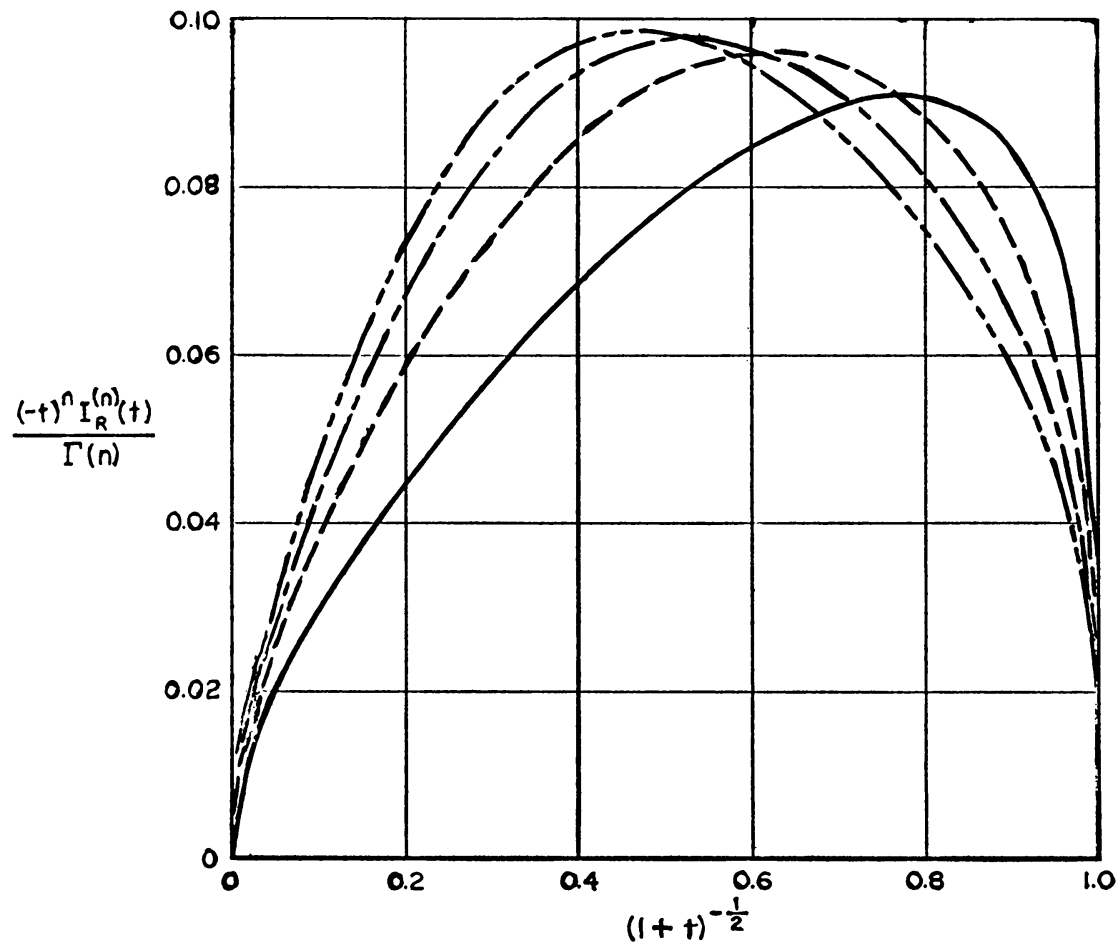

FIg. 2. Derivatives of Ramanujan's integral. $n=1:-; n=2:-\cdots ; n=3:--$ - $;$ $n=4$ : - 


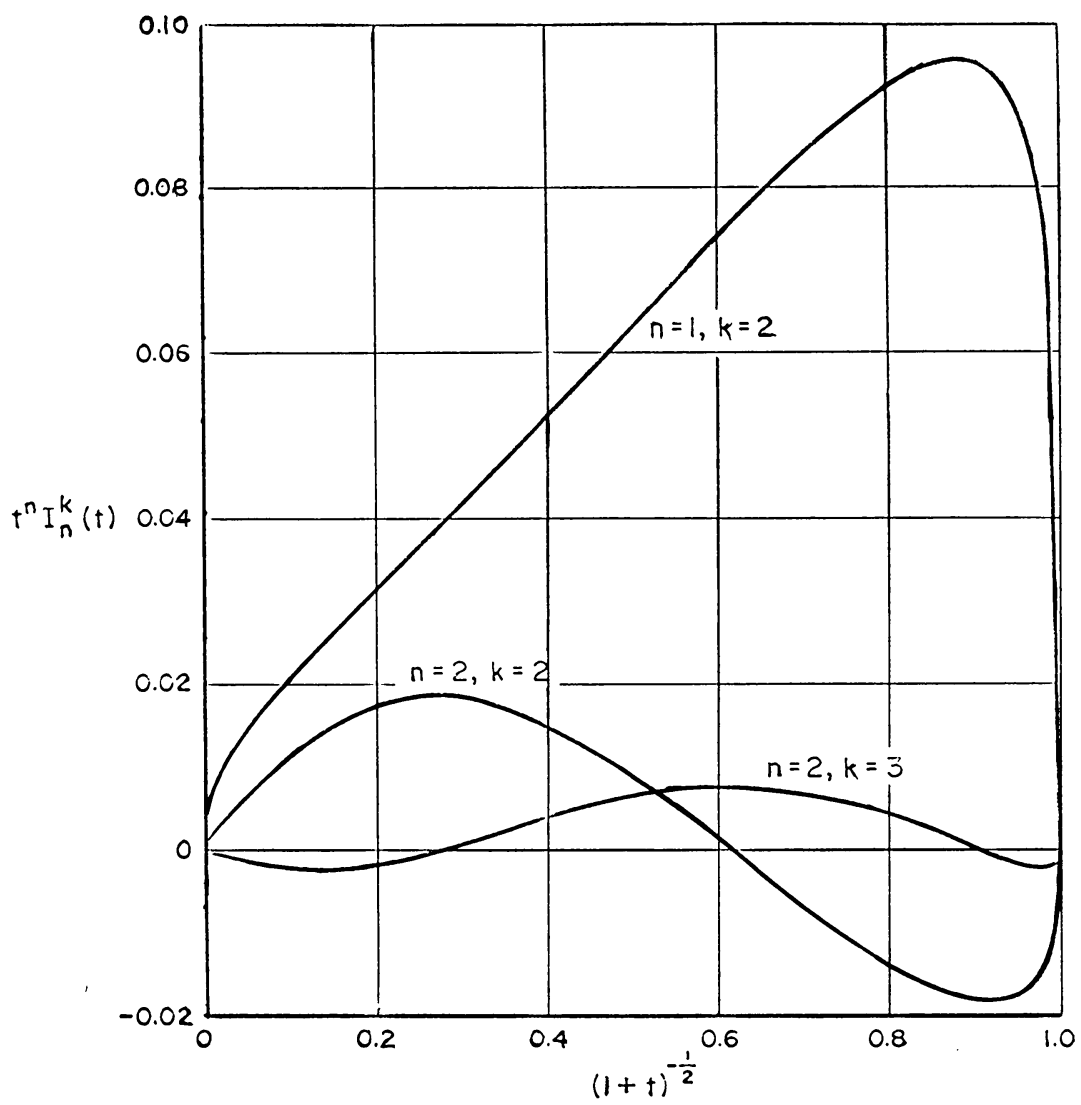

FIG. 3. Some of the contour integrals $I_{n}^{k}(t)=(2 \pi i)^{-1} \int_{-\infty}^{(0+)} e^{z t} z^{n-1}(\ln z)^{k} d z$.

TABLE II

Parameters in approximation to $I_{R 2}+I_{R 3}, E q$. (7)

\begin{tabular}{c|c}
\hline$u_{0}$ & .12943 \\
\hline$a_{0}$ & .07470061 \\
$a_{1}$ & 3.89739242 \\
$a_{2}$ & .01585440 \\
$a_{3}$ & .002866198 \\
\hline$b_{0}$ & 2.97733011 \\
$b_{1}$ & 8.86799361 \\
$b_{2}$ & 1.0 \\
\hline
\end{tabular}

where $u=\ln (1+t)$ and the parameters are given in Table II, with an error $<.0005$ in absolute magnitude over the range $.003 \leqq t \leqq \infty$. For $t>5$, the magnitude of the error is $<.000025$. The error at the origin is .00254 . This result was obtained using a slight modification of Stoer's direct method [9]. It should not be considered a best fit in the Chebyshev sense, though, as the error curve is of far from 
standard form. Similar approximations may be obtained for the higher-order functions $t^{n} I_{R}{ }^{(n)}$, but none will be presented here since these functions can be computed about as easily by Gauss-Laguerre quadrature, as previously described.

6. Acknowledgment. I should like to thank Dr. R. P. Kenan for his assistance and suggestions concerning ('omputer programming and other aspects of this work.

Battelle Memorial Institute

Columbus, Ohio

1. H. S. Carsla $\&$ J. C. Jaeger, Conduction of Heat in Solids, 2nd ed., Oxford Univ. Press, New York, 1959, pp. 339-341.

2. R. H. Ritchie \& A. Y. SaKaklra, "Asymptotic expansions of solutions of the heat conduction equation in internally bounded cylindrical geometry," J. A ppl. Phys., v. 27, 1956, pp. $1453-1459$. MR 18, 780.

3. L. V. Spencer \& U. Fano, Phys. Rev., v. 93, 1954, pp. 1172-1181.

4. (․ H. Hardy, Ramanujan, Cambridge Univ. Press, Cambridge; Macmillan, New York, 1940, pp. 195-198. MR 2, 210.

5. V. Volterra, Atti. Accad. Lincei Mem. Cl. Sci. Fiz. Mat. Nat., v. 11, 1916, pp. 167-249.

6. A. Erdélyi, W. Magnus, F. Oberhettinger \& F. G. Tricomi, Higher Transcendental Functions, Vol. III, McGraw-Hill, New York, 1955, pp. 217-224. MR 16, 586.

7. A. Erdélyi, W. Magnus, F. Oberhettinger \& F. G. Tricomi, Higher T'ranscendental Functions, Vol. I, Me(iraw-Hill, New York, 1953, p. 266. MR 15, 419.

8. P. R A B INOWITZ \& G. WEISS, "Tables of abscissas and weights for numerical evaluation of integrals of the form $\int_{0}^{\infty} e^{-x} x^{n} f(x) d x$, "MTAC, v. 13, 1959, pp. 285-294. MR $21 \# 6713$.

9. J. STOER, "A direct method for Chebyshev approximation by rational functions," J. Assoc. Comput. Mach., v. 11, 1964, pp. 59-69. MR 29 \#1479.

\section{Note on the Calculation of Fourier Series}

\section{By Philip Rudnick}

Cooley and Tukey have recently presented an algorithm for the machine calculation of Fourier series [1]. In this connection mention should be made of the similar method described by Danielson and Lanczos [2]. Although the latter is less elegant and is phrased wholly in terms of real quantities, it yields the same results as the binary form of the Cooley-Tukey algorithm with a comparable number of arithmetical operations.

A small-computer program has been written in this laboratory which uses the Danielson-Lanczos method with one minor modification, described below. ${ }^{*}$ In this form cosine and sine series may be evaluated independently of one another, and as with the Cooley-Tukey process, the calculation can be performed by replacing input data with results, without any substantial storage requirement beyond that set by the original number of input coefficients. The procedure used is readily extensible to the computation of $[(N / 2)+1]$ cosine and $[(N / 2)-1]$ sine sums, from an original sequence of $N$ real numbers, where $N$ is any power of 2 greater than the fourth. Two executions of this procedure will yield values for a set of $N$ complex Fourier series of $N$ terms each, and can be accomplished by $N\left[\log _{2} N+3\right]$ real multiplications and $7 N / 2\left[\log _{2} N-23 / 7\right]$ real additions. For the same task the

Received December 29, 196:).

* Work of the laboratory is sponsored by the Office of Naval Research. 